\title{
Auf der wissenschaftsgeschichtlichen Agenda: Breslauer Germanistik zwischen 1811-1918
}

\author{
Wojciech Kunicki: Germanistische Forschung und Lehre an der König- \\ lichen Universität zu Breslau von 1811 bis 1918. Unter besonderer Berück- \\ sichtigung der Studien zur neueren deutschen Literatur- und Kulturge- \\ schichte, Leipziger Universitätsverlag, Leipzig 2019, 422 S.
}

https://doi.org/10.19195/0435-5865.146.11

Die Geschichte der Germanistik ist in den letzten Dezennien stark ins Zentrum des wissenschaftsgeschichtlichen Interesses gerückt, sodass man in diesem Bereich von einer Art memory boom sprechen kann. Die wissenschaftshistorische Germanistik-Agenda weist unzählige kenntnis- und gedankenreiche Arbeiten auf: Monographien und grundlegende Sammelbände, umfassende Abrisse, wissenschaftliche Aufsätze, aber auch Mitteilungen, die das kommunikative Netzwerk und Positionierungen im Gelehrtenmilieu, biographische und institutionsgeschichtliche, ja alle möglichen diversen wissenschaftsgeschichtlichen Aspekte mit besonderer Berücksichtigung der inhaltlichen Neuansätze ans Tageslicht heben.

Diese Forschungswelle hat ebenso mehrere Studien über die Breslauer Germanistik hervorgebracht: Die Rede ist sowohl von Überblicksdarstellungen als auch von Studien zu den einzelnen Breslauer Germanisten. Gemessen an der Relevanz Breslaus als germanistische Forschungs- und Lehrstätte hat sie bislang eine adäquate Beachtung gefunden. Es geht hier nämlich um ein einmaliges wissenschaftsgeschichtliches Phänomen.

Die Vertreter der Breslauer Germanistik, deren Anfänge auf das Jahr 1811 zurückzuführen sind, haben nicht nur in der Aufbauphase dieser Disziplin eine nicht zu unterschätzende Rolle gespielt, sondern sind illustre Namen der deutschen Germanistik bis 1945. Nachdem aus Breslau nach dem Zweiten Weltkrieg Wrocław, eine Stadt in Westpolen, geworden war, entwickelte sich die Germanistik an der polnischen Alma Mater Wratislaviensis zu einem der weltweit wichtigsten Zentren der Auslandsgermanistik. Dieses einzigartige Beziehungsgeflecht, dessen Eckpunkte durch Preußen, das Deutsche Kaiserreich, die Weimarer Republik, das Dritte Reich und, nach dem Bruch des Jahres 1945, durch Polen gekennzeichnet sind, wurde 2011 während eines Jubiläumskongresses anlässlich des 200jährigen Bestehens der Breslauer Germanistik thematisiert. ${ }^{1}$

Von mehreren polnischen und deutschen Autoren, die die Geschichte und Gegenwart der Deutschen Philologie in Wrocław unter die Lupe genommen haben, soll an erster Stelle Professor Wojciech Kunicki, Leiter des Lehrstuhls für deutsche Literatur von ihren Anfängen bis 1830 an der Universität Wrocław, genannt werden, der die Thematik der deutschen und polnischen Germanistik in der Oderstadt in seine zahlreichen Publikationen, Seminare und Vorträge aufgenommen hat. Es seien hier wenigstens seine zwei monographischen Studien in Buchform genannt, von denen der Band über die Breslauer Germanistik im Zeitraum zwischen 1811-1918 im Rahmen eines Prestigeprojektes entstand, das vom Nationalen Zentrum für Wissenschaft der Republik Polen finanziert, und 2020 mit

\footnotetext{
1 Tomiczek 2013: 17-23.
} 
dem Leopoldina-Forschungspreis der Deutsch-Polnischen Gesellschaft der Universität Wrocław ausgezeichnet wurde. ${ }^{2}$

Angesichts der hier bereits angedeuteten, relativ umfangreichen Forschungsliteratur zur Germanistik im 19. Jahrhundert - zu nennen sind etwa die Studien von Lothar Bluhm, Jürgen Fohrmann, Ulrich Hunger, Rainer Kolk, Uwe Mewes und Wilhelm Voßkamp -, wie auch im Zuge mehrerer Arbeiten zu einigen Vertretern der Breslauer Germanistik, u.a. zu Friedrich Heinrich von der Hagen, Johann Gustav Gottlieb Büsching, August Heinrich Hoffmann von Fallersleben und Gustav Freytag, schloss sich Wojciech Kunicki an den gegenwärtigen Kenntnisstand an und setzte es sich zum Ziel, ihn durch Erschließung bislang brachliegender Archivmaterialien und mehrerer, eigentlich vergessener Veröffentlichungen der Breslauer Germanisten zu bereichern, was er in der Einleitung seines Werkes Germanistische Forschung und Lehre an der Königlichen Universität zu Breslau von 1811 bis 1918. Unter besonderer Berücksichtigung der Studien zur neueren deutschen Literaturund Kulturgeschichte festhielt:

Das Ziel der vorliegenden Arbeit ist nicht eine lückenlose Geschichte der Germanistik an der königlichen Universität zu Breslau in der Zeit von 1811 bis 1918 zu schreiben, sondern eher vor dem Hintergrund der skizzierten geschichtlichen Hauptlinien, insbesondere die Problematik der Studien zur neueren deutschen Literatur an der Breslauer Universität zu erörtern. Der Verfasser ist vor allem darum bemüht, eine gesicherte Grundlage für seine Reflexionen zu schaffen, deshalb spielen hier die Dokumente eine wesentliche Rolle. Der Autor knüpft selbstverständlich an die bereits bestehende umfangreiche Forschungsliteratur zur Germanistikgeschichte auch an der Universität Breslau an. Deshalb sind die Kapitel zu Friedrich Heinrich von der Hagen, Johann Georg [soll sein: Gustav - M.H.] Gottlieb Büshing oder August Heinrich Hoffmann von Fallersleben entweder auf einige Aspekte ihrer vor allem methodischen Positionierung oder auf ihre Beschäftigung mit der neueren deutschen Literatur fokussiert. In methodischer Hinsicht ist die vorliegende Abhandlung auf Persönlichkeiten konzentriert, die das Fach Germanistik auch in Breslau geprägt haben. Hinter persönlichen Konstellationen verbergen sich oft auch institutionelle, weltanschauliche und politische Hintergründe, die hoffentlich in der Darstellung zum Vorschein kommen. Der vorliegende Band knüpft an die 2002 erschienene Monographie über die Breslauer Germanistik von 1918 bis 1945 an. (S. 11)

Als erfahrener Autor geht Kunicki in seinem Buch also vom Bekannten aus, um Terra incognita zu erschließen. Dazu hat er sein eigenes Analysewerkzeug erarbeitet, d.h. er erhebt keinen Anspruch auf eine nach Vollständigkeit strebende Gesamtdarstellung, sondern navigiert - stets an die bestehende Sekundärliteratur anschließend - die von ihm aufgefundenen Dokumente und wissenschaftlichen Publikationen der Breslauer Germanisten. Diese Verfahrensweise markiert eine wegweisende Position: Die Studie ist für ein Fachpublikum vorgesehen, dem das Sujet im Allgemeinen bereits bekannt ist. Dieses Fachpublikum ist dann imstande, durch die Funde Kunickis einzelne Aspekte aus neuen Perspektiven wahrzunehmen.

Hervorzuheben ist der gewaltige Umfang der Materialbasis, auf welcher der Autor neue Facetten der germanistischen Landschaft Breslaus in der Zeit von 1811-1918 aufzudecken weiß: In allererster Reihe handelt es sich um eine Vielzahl von Dokumenten aus dem Geheimen Staatsarchiv Preußischer Kulturbesitz Berlin-Dahlem, denen zahlreiche Quellen aus den Archiven und Bibliotheken in Berlin, Marburg, Weimar, Leipzig, Wien

${ }^{2}$ Kunicki 2019 (Seitenangaben aus diesem Buch werden im vorgelegten Text in runden Klammern gemacht). 
und Wrocław folgen (sie alle sind am Ende des Buches aufgelistet). Es geht u.a. um private, Fach- und Dienstkorrespondenz, Tagebucheintragungen und Vorlesungsverzeichnisse, die sich auf diverse Felder beziehen: germanistische (sprach-, literatur- und kulturgeschichtliche) Einzelthemen und Auseinandersetzungen (Konkurrenz- bzw. Machtkampf), Karrierebestrebungen, kollegiale Sympathien und Verfeindungen, Bildungs- und Kulturpolitik (in Forschung und Lehre), organisatorische Angelegenheiten und Institutionalisierungsprozesse. Hier ein aussagekräftiges Beispiel: Obwohl die Forschung sich in den letzten Jahren auf Gustav Freytag, den bekannten Breslauer Privatdozenten, eingelassen hat, ${ }^{3}$ ist das präzise Verzeichnis seiner Lehrveranstaltungen mit der jeweils genauen Zuhörerzahl Wojciech Kunicki zu verdanken. Er hatte selbiges im Geheimen Staatsarchiv Preußischer Kulturbesitz in Berlin-Dahlem ausfindig gemacht. Alles in allem stößt der Leser auf einen äußerst umfangreichen Stoff, der ihm Zugang zu Biographien der einzelnen Gelehrten wie auch zu jenem Spannungsfeld ermöglicht, auf dem sich das germanistische Leben Breslaus etablierte und entwickelte. Da meldet sich die Vorliebe Kunickis zum Detail zu Wort, seine Untersuchung wird immer wieder durch die bislang unbekannten und relevanten Tatsachen aus den angeführten Dokumenten verlebendigt.

Die Struktur des Buches basiert auf einer chronologischen Auflistung der Professoren der Breslauer Alma Mater, die in ihrer akademischen Tätigkeit (Forschung und Lehre) das germanistische Pensum realisiert haben, so u.a. Friedrich Heinrich von der Hagen, Johann Gustav Gottlieb Büsching, August Kahlert, August Heinrich Hoffmann von Fallersleben, Theodor Jacobi, Karl Weinhold, Gottschalk Eduard Guhrauer, Friedrich Wilhelm Pfeiffer, Felix Bobertag, Max Koch, Friedrich Vogt und Theodor Siebs. Von der Prämisse ausgehend, Neues zum bisherigen Kenntnisstand aufgrund der ausgesuchten Funde beizutragen, werden im besprochenen Buch bei den behandelten Autoren neue Erkenntnisse in unterschiedlichem Ausmaß erbracht. Dies hatte gewisse „Asymmetrien“ im Umfang der einzelnen Kapitel/Passagen zur Folge, wenn man z. B. die fast acht Seiten, die dem wohl berühmtesten Breslauer Germanistikprofessor Hoffmann von Fallersleben gewidmet wurden, mit der ausführlichen Darstellung z.B. Eduard Guhrauers (33 Seiten) vergleicht. Daraus darf aber kein Vorwurf gemacht werden (!), ganz im Gegenteil: Bei dem breiten thematischen Spektrum der Abhandlung konnte - wie bereits gesagt - keine Komplexität angestrebt, sondern sollte dem interessierten Publikum ein Einblick in die neuen Details ermöglicht werden. So stellt der relativ kurze Beitrag über Hoffmann von Fallersleben einen Anschluss an die seit drei Jahrzehnten besonders intensive Forschung zum Autor des Liedes der Deutschen dar, die dem polnischen Wissenschaftler selbstverständlich bestens bekannt ist und in seinem Buch in den Anmerkungen angeführt wird. Am Fall Hoffmann von Fallersleben lässt sich prägnant die Strategie des Autors aufzeigen, punktuelle Ergänzungen und Revisionen vorzunehmen. Dies ist auf die imponierende Fülle des von Wojciech Kunicki erschlossenen Quellenmaterials zurückzuführen, die seinen Darstellungen zugrunde liegt. Wie bereits gesagt, ist nicht die Komplexität das Hauptziel des Autors, sondern die Anstrengung, der Fachwelt neue, bislang unbekannte quellengestützte Funde näherzubringen.

$\mathrm{Zu}$ diesen Funden gehören neben den zahlreichen Details sowohl wissenschaftliche als auch populärwissenschaftliche Veröffentlichungen, die in der bisherigen Forschung

3 Dazu siehe die Arbeiten von Bernt Ture von zur Mühlen, u.a. sein Aufsatz: Gustav Freytags Breslauer Jahre 1835 bis 1846. In: Hałub / Mańko-Matysiak 2016: 218-248. 
entweder kaum wahrgenommen wurden oder lediglich peripher auftauchten. Dieser für den Band charakteristische Neuansatz ist im Falle der Abhandlung über den Vater der Breslauer Germanistik, Friedrich Heinrich von der Hagen, deutlich sichtbar, der sich - wie bekannt - einen Namen als Herausgeber des Nibelungenliedes und anderer Werke aus der altdeutschen Literatur und Kunst gemacht hat, worüber z.B. Eckhard Grunewald mit größter Sachkenntnis in seiner monographischen Gesamtdarstellung berichtet hat. ${ }^{4}$ Die Vorliebe von der Hagens für das Mittelalter weist auch - was der polnische Autor im Anschluss an die Studie Grunewalds ans Tageslicht zu heben weiß -Berührungspunkte zu Schlesien auf; eine Tatsache, die bislang lediglich am Rande erwähnt wurde. So dreht sich die Behandlung Kunickis um eine Artikelserie von der Hagens Wanderungen durch das alte Breslau und dessen weitere schlesienbezogene Pressebeiträge, wodurch der Blick auf den bekannten Protogermanisten an der Alma Mater Wratislaviensis wesentlich erweitert wird. Die Schlesienforschung wurde bei der in diesem Buch vorgenommenen Neukontextualisierung des Euvres der Breslauer Germanisten zu einem wichtigen Leitfaden, da es eine ganze Reihe von Einzelbeobachtungen zum breitgefächerten schlesischen Szenario im wissenschaftlichen Vorgehen der präsentierten Breslauer Germanisten aufweist.

Nicht nur in dem im Buch behandelten Zeitraum markierten die Schlesienforschungen ein wichtiges Spezifikum der Breslauer Germanistik, auch in der darauffolgenden deutschen Zeit haben sich Breslauer Germanisten intensiv mit der schlesischen Kulturgeschichte auseinandergesetzt. Die Erforschung des Kulturraumes Schlesien blieb gleichermaßen in der polnischen Gegenwart nach $1945 \mathrm{im}$ germanistischen Milieu an der Alma Mater Wratislaviensis ein leitendes Desiderat; diesbezüglich haben polnische Gelehrte im Bereich der Erforschung des Schlesienlandes bedeutende Leistungen hervorgebracht. Alles in allem ist die Schlesienforschung zu einem Signum der über 200 Jahre zählenden Breslauer Germanistik geworden. ${ }^{5}$

Mit der Fülle des neuen Materials wollte Wojciech Kunicki zum ersten Mal in der bisherigen wissenschaftsgeschichtlichen Forschung den Übergang der Breslauer Germanistik von den altdeutschen Studien hin zur Untersuchung der neueren deutschen Literatur und Kultur nachzeichnen, was dem Autor sogar als Hauptziel seiner Arbeit vorschwebte. Mehrere Jahrzehnte hindurch orientierte sich insbesondere die Aufbauphase der Germanistik an dem altdeutschen Topos, galt sogar als ,,antiquarische Wissenschaft“, der es an auf neuere deutsche Literatur- und Kulturgeschichte fokussiertem Zusammenhang fehlte. Wojciech Kunicki ist es gelungen, dieses schwer zu ordnende Feld auszuarbeiten und zu beweisen, dass Breslau hier wirklich eine Vorreiterrolle spielte (S. 378). Der Rekurs auf die neuen Positionen der Breslauer Germanisten hat einen prägenden Einfluss auf das Narrativ des Buches, im Hinblick darauf folgt der Autor mit Detektivsinn den auf die neuere deutsche Literatur fokussierten Recherchen der Breslauer Germanistik. So ist es nicht verwunderlich, dass z.B. den Versuchen des bisher verkannten Felix Bobertags, „die neue deutsche Literatur am Germanistischen Seminar zu etablieren" (S. 282), eine ausführliche Passage gewidmet wurde. Kunicki analysiert die Strategien der einzelnen Literaturwissenschaftler anhand ihrer Werke, aber auch die institutionellen Strategien anhand der von ihm

4 Grunewald 1988.

5 Im Rahmen des bisher letzten, 4. Kongresses der Breslauer Germanistik haben die Germanisten von der Universität Wrocław über Breslau als europäisches Kulturphänomen in polnischer Sprache referiert, um dem polnischen Publikum das relativ wenig bekannte plurikulturelle Erbe der schlesischen Metropole näher zu bringen. Siehe dazu den Tagungsband: Hałub 2017. 
gesammelten Quellenmaterialien, um hier exemplarisch die universitäre Fachkorrespondenz anzuführen, in der auf „die Notwendigkeit“ hingewiesen wurde, „Lehrstühle für neuere deutsche Literatur einzurichten“" (S. 292). Vor dem institutionellen Hintergrund werden Profilierungen der einzelnen Forscher sorgfältig auf Basis des aufgefundenen Materials ausgearbeitet. Beispielsweisewären hier die Positionsbestimmungen zu nennen, die auf den bisher zu Unrecht peripher behandelten Gottschalk Eduard Guhrauer im Kontext der „Vorstöße in die Interdisziplinarität“ (S. 149) zurückzuführen sind, oder auch das vielfältige, auf die neuere deutsche Literatur und Kultur profilierte Forschungsspektrum Max Kochs (u.a. seine Auseinandersetzung mit Karl Emil Franzos über die Feindschaft zwischen den schwäbischen Romantikern und Heine oder seine Studien zu Richard Wagner), einer der Koryphäen der Breslauer Germanistik, dem knapp 50 Seiten gewidmet wurden.

Zusammenfassend ist festzuhalten: Als profunder Kenner der Materie hat Wojciech Kunicki, dank seines immensen Aufwandes, den von ihm gesammelten umfangreichen Quellenstoff nicht nur im wissenschaftsgeschichtlichen (vor allem literaturhistorischen) Rahmen, sondern auch vor dem kulturpolitischen Hintergrund präzise situiert und interpretiert. Deswegen ist sein enormer analytischer Sammlungs- und Rekonstruktionsaufwand für das Fachpublikum aus zweierlei Gründen nützlich: Mit zahlreichen neuen Quellenfunden hat er eine solide Grundlage für die weitere Rekonstruierung der germanistischen Forschung und Lehre an der Breslauer Universität, von ihrer Geburtsstunde im Jahre 1811, über den institutionellen Aufbauprozess und die darauffolgende erfolgreiche Etablierungsphase bis 1918,geschaffen. Dabei hat er vor allem literaturhistorische Positionierungen von Breslauer Germanisten und dadurch das von ihnen gestaltete germanistische Profil der Odermetropole stringent herausgearbeitet. Ein ansehnlicher gewinnbringender Mehrwert für die Wissenschaftsgeschichte.

\section{Literatur}

Behr, Hans-Joachim / Blume, Herbert / Rose, Eberhard (Hrsg.) (1999): August Heinrich Hoffmann von Fallersleben 1798-1998. Festschrift zum 200.Geburtstag. Bielefeld. Hier: Teil III: Hoffmann als Germanist: Facetten eines Berufslebens [Sieben Aufsätze zu verschiedenen Aspekten seiner Tätigkeit als Germanist - M.H.]. (Braunschweiger Beiträge zur deutschen Sprache und Literatur, Bd. 1).

Fohrmann, Jürgen / Voßkamp, Wilhelm (Hrsg.) (1994): Wissenschaftsgeschichte der Germanistik im 19. Jahrhundert. Stuttgart/Weimar.

Grunewald, Eckhard (1988): Friedrich Heinrich von der Hagen 1780-1856. Ein Beitrag zur Frühgeschichte der Germanistik. Berlin/New York.

Hałub, Marek (Hrsg.) (2017): Wroclove. Fenomen kultury europejskiej. Wrocław.

Hałub, Marek / Mańko-Matysiak, Anna (Hrsg.) (2016): Śląska Republika Uczonych. Schlesische Gelehrtenrepublik. Slezská vedécká obec, Bd. 7. Dresden/Wrocław.

Kunicki, Wojciech (2002): Germanistik in Breslau von 1918 bis 1945. Dresden.

Kunicki, Wojciech (2019): Germanistische Forschung und Lehre an der Königlichen Universität zu Breslau von 1811 bis 1918. Unter besonderer Berücksichtigung der Studien zur neueren deutschen Literatur- und Kulturgeschichte. Leipzig.

Kunicki, Wojciech / Zybura, Marek (Hrsg.) (2011): Germanistik in Polen. Zur Fachgeschichte einer literaturwissenschaftlichen Auslandsgermanistik - 18 Porträts. Osnabrück. 
Kunicki, Wojciech / Zybura, Marek (Hrsg.) (2015/2016): Geschichte der literaturwissenschaftlichen Germanistik in Polen. Bd. 1: Inhalte und Methoden. Leipzig 2015; Bd. 2: Kulturpolitik und Kulturtransfer. Leipzig 2016.

Meves, Uwe (2004): Ausgewählte Beiträge zur Geschichte der Germanistik und des Deutschunterrichts im 19. und 20. Jahrhundert. Hildesheim (Spolia Berolinensia, Bd. 24).

Tomiczek, Eugeniusz (2013): 200 Jahre Breslauer Germanistik. In: Bartoszewicz, Iwona / Hałub, Marek / Tomiczek, Eugeniusz (Hrsg.): Sprache-Literatur-Kultur im germanistischen Gefüge, Bd. 1. Wrocław/Dresden.

Marek Hatub

(Universität Wrocław, Wrocław)

ORCID: 0000-0002-4217-2527

Marek Hałub, Uniwersytet Wrocławski, Instytut Filologii Germańskiej, pl. biskupa Nankiera 15b, 50-140 Wrocław, Polen, E-Mail: marek.halub@uwr.edu.pl.

Received: 10.07.2020, accepted: 1.03.2021

\begin{abstract}
Alice Cadeddu, Claudia Junk, Thomas F. Schneider (Hrsg.): Weltweit Worldwide - Remarque. Beiträge zur aktuellen internationalen Rezeption von Erich Maria Remarque, V\&R unipress, Göttingen 2020, 268 S.
\end{abstract}

https://doi.org/10.19195/0435-5865.146.12

$\mathrm{Zu}$ seinen Lebzeiten zählte Erich Maria Remarque zu den wirkmächtigsten deutschsprachigen Autoren. Generationen der Leser in der ganzen Welt galt und gilt er hauptsächlich als Verfasser des Bestsellers Im Westen nichts Neues, der ihm zwar Lob der Leserschaft brachte, aber ihn zugleich scharfer Kritik der politischen Gegner aussetzte. Während die Verfilmung des Romans in Hollywood mit einem Oscar ausgezeichnet wurde, stempelte man das Werk im nationalsozialistischen Deutschland als „schädliches und unerwünschtes Schrifttum“ ab. Die feindliche Einstellung der Parteiführer kann man allerdings nachvollziehen. Remarque habe doch in seinen Romanen ,ein gewaltiges Sittengemälde seiner Zeit geschaffen, einer Epoche, die durch Krieg, Terror, Vertreibung, Gewalt und Lüge beherrscht war und in der menschliche Werte und Qualitäten, Liebe und Freundschaft, stets bedroht blieben“ (W.v. Sternburg). Mit seinem Werk ist er zum Chronisten der deutschen Geschichte in der ersten Hälfte des zwanzigsten Jahrhunderts geworden.

Im Laufe seiner schriftstellerischen Karriere entpuppte sich Remarque als eleganter Weltbürger, höchst aktiver Partygast und leidenschaftlicher Liebhaber. Sein Alltag war geprägt durch Luxus, zahllose Liebesaffären und Alkoholexzesse. Aber nicht der rauschhafte Lebensstil sicherte ihm den Platz in der Geschichte der Weltliteratur, sondern vielmehr die in seinen Büchern angesprochenen Themen und Motive, darunter - um nur einige zu nennen - Migration, Flucht, Identität, Unterdrückung, Empathie, Pazifismus, Zivilisation bzw. Humanität. Die Frage nach deren Aktualität sowie nach dem internationalen Stellenwert des Schriftstellers und seines Schaffens 50 Jahre nach dem Tod versucht der 2020 in Vandenhoeck \& Ruprecht erschienene Band zu beleuchten. Seine Herausgeber sind Mitarbeiter des 